\title{
Prevention of Venous Thrombotic Events in Brain Injury: Review of Current Practices
}

\author{
Stuart Glassner, D.O., Karan Srivastava, Paul Cofnas, Brian Deegan, Peter \\ DeMaria, Rimsky Denis, and Enrique Ginzburg, M.D.* \\ Jackson Memorial Hospital and University of Miami, Florida, USA
}

\begin{abstract}
Venous thromboembolic event after traumatic brain injury represents a unique clinical challenge. Physicians must balance appropriate timing of chemoprophylaxis with risk of increased cerebral hemorrhage. Despite an increase in the literature since the 1990s, there are clear disparities in treatment strategies. This review discusses the prominent studies and subsequent findings regarding the topic with an attempt to establish recommendations using the existing evidence-based literature.
\end{abstract}

KEY WORDS: Deep venous thrombosis, prophylaxis, traumatic brain injury, venous thromboembolic events

\section{INTRODUCTION}

Despite multiple studies investigating prevention of venous thromboembolic events (VTE) with pharmacologic or mechanical strategies for brain trauma patients, there still exists much variability in actual practice. The discrepancy arises in the recognition of intracranial bleeding risk and thereby the appropriate timing of intervention. Due to a paucity of randomized control trials, The Brain Trauma Foundation (2007) offers only level III evidence as expert consensus in recommending the use of VTE prophylaxis with low-dose heparin (unfractionated heparin) or low-molecular-weight heparin (LMWH)

\footnotetext{
Abbreviations: DVT, deep venous thrombosis; GCS, Glasgow coma score; ICH, intracerebral hemorrhage; LMWH, lowmolecular-weight heparin; PE, pulmonary embolism; PT, prothrombin time; PTT, partial thrombin time; SAH, subarachnoid hemorrhage; TBI, traumatic brain injury; TF, tissue factor; UFH, unfractionated heparin; VTE, venous thromboembolic events.

Citation: Glassner S, Srivastava K, Cofnas P, Deegan B, DeMaria P, Denis R, Ginzburg E. Prevention of Venous Thrombotic Events in Brain Injury: Review of Current Practices. RMMJ 2013;4 (1):eooo1. doi:10.5041/RMMJ.10101

Copyright: (C) 2013 Glassner A, et al. This is an open-access article. All its content, except where otherwise noted, is distributed under the terms of the Creative Commons Attribution License (http://creativecommons.org/licenses/by/3.o), which permits unrestricted use, distribution, and reproduction in any medium, provided the original work is properly cited.

Conflict of interest: No potential conflict of interest relevant to this article was reported.

* To whom correspondence should be addressed. E-mail: eginzburg@miami.edu
} 
in patients with severe traumatic brain injury (TBI). ${ }^{1}$ They give caution due to risk of hemorrhagic expansion but no guideline regarding initiation of treatment or agent selection. This review discusses the prominent studies and subsequent findings regarding the topic, with an attempt to establish recommendations using the existing evidence-based literature.

\section{INVESTIGATIONAL STUDIES}

The body of related literature provides several assumptions followed in this paper for consistency. First are the standard anatomical location definitions for deep venous thrombosis (DVT) and proximal DVT that risk propagation to a pulmonary embolism (PE) which may necessitate a more aggressive intervention. The second accepted assumption is based on the fact that most studies cited below include routine use of mechanical prophylaxis alone or in combination with drug prophylaxis. Prior investigations, most recently by Ekeh et al., ${ }^{2}$ concluded that physical compression by itself is inadequate to prevent DVT. Finally, standard screening methods for VTE include ultrasound venous Doppler unless noted otherwise, although venogram was the gold standard for diagnosis in early research studies.

VTE as consequence of trauma was formally quantified in 1994 by Geerts et al. in an extensive study published in the New England Journal of Medicine. 3 This prospective study had inclusion of only severe trauma victims (using a cut-off of $>9$ on the Injury Severity Score) and did not utilize any VTE prophylaxis during patient course. Instead, a predominance of the 349 patient sample underwent contrast venography $1-3$ weeks after admission. The authors found an overall incidence of $57.6 \%$ (95\% CI $52 \%-62.8 \%$ ) incurring DVT and $18.1 \%$ with proximal DVT, despite only $1.5 \%$ of patients displaying clinical symptoms. ${ }^{3}$ Further stratification of injury based on major location (head versus spine versus lower limbs versus face/chest/abdomen) and type yielded a framework for epidemiologic difference in risks. Subgroup analysis revealed that of 91 patients with head trauma alone or with other injury, 53.8\% presented with distal deep vein clots and $19.8 \%$ presented with proximal (thigh) deep vein clots, for a total of $73 \%$ of this population. It is important to note that of the 51 patients with head trauma as their only injury, the incidence of DVT was $39 \%$. As expected, head trauma with lower extremity trauma experienced a DVT rate of $77 \%$, and the study found femur or tibial fractures were an independent risk factor, along with spinal cord injury, age, surgery, and blood transfusion. ${ }^{3}$ Head injuries were grouped as a whole and not further divided.

TBI-induced coagulopathy contributes risk to this population. A review by Laroche et al. from June 2012 characterizes the phenomenon as a combination of both hypercoagulable and hypocoagulable states. 4 It is hypothesized that the bleeding diathesis is rooted in the elevated release of brain and systemic tissue factor (TF) due to injury. The resulting over-stimulation of the coagulation cascade can then foster a consumptive coagulopathy, though other mechanisms are being investigated.4 Additionally, in 2007 Nekludov et al. further found evidence of TF-triggered hyperfibrinolysis as well as a hypercoagulability with generation of micro-thrombosis. 5 In a related review of TBI patients, evidence of hemorrhagic progression or new development of ischemic lesions after initial emergency room presentation was found in $85 \%$ of patients with laboratory evidence of coagulopathy on admission, as compared to $31 \%$ of those with normal levels. 5 Thus in a variety of analyses, diminished platelet counts, increased partial thrombin time (PTT), and elevated prothrombin time are shown to be predictive markers of mortality in severe TBI. Prothrombin time abnormality was specifically noted to be an independent predictor in the landmark multinational IMPACT study (International Mission for Prognosis and Analysis of Clinical Trials in TBI, IMPACT, 2007).5,6

However, due to small sample size, Geerts' earliest paper was not able to state whether brain injury itself is an independent risk factor for thromboembolic events. 3 Consequently, a flurry of investigational studies appeared in the past few years addressing the question. Most notable was a retrospective study of 2,000 patients by Reiff et al. in 2009.7 It found a $3-4$-fold increase in DVT incidence among TBI patients stratified by "time to initiation" of either low-molecular-weight heparin or low-dose unfractionated heparin. The groups were all on mechanical prophylaxis and varied from $\mathrm{O} \leq$ $24 \mathrm{~h}, 24-48 \mathrm{~h},>48 \mathrm{~h}$, or no intervention given. There was an increased risk throughout the categories, with an absolute risk of $3.6 \%$ in the earliest administration group increasing to $15.4 \%$ at $>48 \mathrm{~h}$. Thus, the study asserts that regardless of timing or use of pharmacologic prophylaxis, TBI counted as a risk but was seen with greater incidence associated with delayed onset of prophylaxis.7 
The Reiff group further consolidated earlier findings, including that of Denson $(2008)^{8}$ and Nathens (2007) 9 among others who strongly hinted at a correlation between VTE and head injury. Denson et al. reported a rate of $25 \%$ in his study. ${ }^{8}$ Furthermore, in 2010 Ekeh et al. analyzed DVT and pulmonary embolism (PE) rates in $677 \mathrm{TBI}$ patients, comparing incidence in isolated head-injured patients with those who had brain trauma combined with extra-cranial damage. ${ }^{2}$ Similar to Geerts, no medical prophylaxis was given, and patients had scheduled screenings for DVT, with Doppler ultrasound. Each patient received compression devices when two lower extremities were viable. The results supported a strong association between VTE and brain trauma. Additionally, both DVT and PE rates were higher with multiple injuries.

In $2009 \mathrm{Kim}$ et al. published an article in Neurocritical Care and found a subtle difference in rates of VTE among patients with subarachnoid hemorrhage, intracerebral hemorrhage, and traumatic brain injury (TBI) with cerebral contusions. ${ }^{10}$ Of the 1,195 patients that met study criteria for inclusion, the incidence of symptomatic VTE for subarachnoid hemorrhage patients, intracerebral hemorrhage patients, and the TBI patients were $6.7 \%, 2.9 \%$, and $3.8 \%$, respectively, resulting in no significant difference. ${ }^{10}$ As in most studies, severity of injury was not an examined variable. The Scales group indirectly investigated the risk of bleeding in various degrees of intracranial hemorrhage using a decision-point model for medical prophylactic use after 24 hours. ${ }^{11}$ They found that no difference surfaced in rates of DVT regardless of severity in intracranial hemorrhage up to the first 24-hour time-frame, though it is conceivable from earlier studies that this would change as the number of hospital days increased.

The crux of the issue, however, involves the timing of prophylactic intervention. This continues as a provocative issue in the preventative treatment of VTE. It is not uncommon for significant variation to occur even in the same institution as illustrated by an earlier study by Scales et al. ${ }^{12}$ Their 2009 survey questioned 160 Canadian neurocritical care physicians (some at the same hospital) and found anything but a consensus on initiation of pharmaceutical prophylaxis. This followed Carlile in 2006 who published the results of an intensive multicenter survey of US practice patterns involving VTE screening and prophylaxis after TBI in the acute care setting. ${ }^{13}$ The data suggested inconsistent use of drug prophylaxis (56\%) with more common utilization of mechanical device (69\%) in these patients. This lack of consensus echoes findings in other reports that mention a scenario akin to "attending-based medicine" whereby use and timing of chemoprophylaxis is subject to physician or surgeon discretion.

A recent Journal of NeuroTrauma article by Dudley et al. may offer insight into the debate. ${ }^{14}$ The study looked at a broader scope of TBI patients and used serial CT scans as a marker of intracerebral hemorrhage stability prior to giving LMWH if no confounding coagulopathy. They chose administration at 48-72 hours, citing prior data that withholding prophylaxis for more than 4 days tripled VTE risk.7,9,15 The population included a spectrum of patients with moderate to severe brain injuries, Glasgow Coma Score varying from 3 to 12, and Injury Severity Score ranging from 4 to 66 . Their results showed overall VTE incidence at $7.3 \%$ with one death resulting from hemorrhagic expansion as revealed by a follow-up CT scan.

It is duly noted that this study had higher rates of VTE than those intervening at 24 hours, which in fact is what the Reiff study (see above) illustrated with its treatment groups receiving prophylaxis at $<24 \mathrm{~h}, 24-48 \mathrm{~h}$, and $>48 \mathrm{~h} .7$ Both papers infer that delays of even 24 hours can contribute to VTE risk. ${ }^{6,14}$ However, this certainly must be balanced with risk of intracerebral hemorrhage expansion, resulting in a risk-to-benefit ratio directing chemoprophylaxis initiation at the 48-72-hour time-frame.

The Dudley study was the first to compare common LMWH agents, enoxaparin and dalteparin, directed by the prior findings by Geerts (1996) who showed a superiority of enoxaparin to unfractionated heparin.14 In the 267-patient retrospective study, the Dudley team found essentially no difference between either LMWH agent in preventing VTE. The investigation did initially reveal a small difference in risk between the two agents; however, the authors cite a negligible discrepancy once baseline characteristics, such as lower starting Glasgow Coma Score in the dalteparin intervention group, were considered. A related 42-month cohort analysis by Minshall et al. in 2011 compared outcome in 386 patients based on type of medical prophylaxis given, but a firm time to initiation of therapy was not delineated. ${ }^{15}$ It inferred patients receiving unfractionated heparin had an 
increased rate of $\mathrm{PE}(3.7 \%)$ against those receiving LMWH (o\%; $P<0.05)$. No hemorrhagic complications occurred in either group. However, the conclusions of this analysis were very limited given that patients with less severe injuries mostly received LMWH, while those with more severe injury were treated with unfractionated heparin. Furthermore, the study had no routine DVT or CT screening and relied solely on clinical judgment versus imaging. ${ }^{15}$

The objection from clinicians about early use of LMWH or unfractionated heparin, within 24 hours, is controversial due to concerns of hemorrhagic extension. However, some reports are worth considering, including those of Norwood et al. ${ }^{16,17}$ Altogether 177 patients were included in this study of whom 150 received enoxaparin at 24 hours after admission for intracerebral hemorrhage or after craniotomy for the injury. CT screening was performed at various intervals including admission, 24 hours after hospitalization, and then after receiving the LMWH. After administration of chemoprophylaxis, $4 \%$ of patients developed further hemorrhage as evidenced on CT head scans.

The study raised the question whether hemorrhagic changes on CT scan are a consequence of the prophylaxis or a natural progression of the TBI. The analysis did not have a control group. ${ }^{16,17}$

\section{CONCLUSION}

Although trauma is a well-established etiology for thromboembolic events, only in the past decade have TBI patients been recognized with an increased risk for VTE. This is most likely due to lengthy hospital immobilization combined with delays in VTE drug regimen prophylaxis. Additionally, the native levels of $\mathrm{TF}$ already residing in the brain increase during injury, along with circulating inflammatory cytokines which favor systemic hypercoagulation. Unfortunately, no significant randomized control trials exist with large patient populations, leaving clinicians with expert consensus and a series of retrospective or prospective studies. At issue is the balance between hemorrhagic extension and VTE risk. These important studies yield information about the highest-risk individuals with inference toward practice parameters. Following these, clinical assessment might appear as follows:
- Establish degree of TBI severity and carry out a survey of additional injuries, especially of the lower extremities.

- Start patient on mechanical compression prophylaxis within 24 hours of admission unless contraindicated.

- As mechanical intervention is insufficient alone, LMWH or unfractionated heparin should be started, if no confounding coagulopathy. Timing of this step is variable and controversial. However, a comfortable balance point between extension of the bleed and VTE risk appears to be 48-72 hours. Again, this is inferred from leading studies and not level I evidence. This differs from the Brain Trauma Foundation which omits timing guidelines. Several studies utilized serial head CTs for bleeding progression both before and after anticoagulant administration.

- The choice of regimen, unfractionated heparin versus LMWH, appears only mildly significant in the brain trauma patient. A number of studies show a superiority of LMWH, but bleeding risks are still a concern $<24$ hours of initiating therapy. Options within LMWH indicate no superiority between enoxaparin or dalteparin, though the q12 hour dosing of the former may prove of greater benefit than daily dosing of dalteparin.

- The predominance of institutions utilized ultrasound venous Doppler in addition to clinical acumen.

In summary, the evidence above has directed the American College of Chest Physicians to recommend the following (2012) ${ }^{18}$ :

- For major trauma patients at high risk for VTE (including brain trauma): mechanical prophylaxis in addition to pharmacologic prophylaxis when not contraindicated by lower-extremity injury.

- For major trauma patients: Use of LMWH or unfractionated heparin or mechanical prophylaxis indicated over no prophylaxis.

- For major trauma patients in whom LMWH and unfractionated heparin are contraindi- 
cated: Use of mechanical prophylaxis indicated over no mechanical prophylaxis.

- For major trauma patients: Use of inferior vena cava filter for primary VTE prevention not indicated.

\section{REFERENCES}

1. Brain Trauma Foundation; American Association of Neurological Surgeons; Congress of Neurological Surgeons; Joint Section on Neurotrauma and Critical Care, AANS/CNS; Bratton SL, Chestnut RM, Ghajar $\mathrm{J}$, et al. Guidelines for the management of severe traumatic brain injury. Deep vein thrombosis prophylaxis. J Neurotrauma 2007;24 (Suppl 1):S32-6.

2. Ekeh AP, Dominguez KM, Markert RJ, McCarthy MC. Incidence and risk factors for deep venous thrombosis after moderate and severe brain injury. $\mathrm{J}$ Trauma 2010;68:912-5.

3. Geerts WH, Code KI, Jay RM. A prospective study of venous thromboembolism after major trauma. $\mathrm{N}$ Engl J Med 1994;331:1601-6. Full Text

4. Laroche M, Kutcher ME, Huang MC, Cohen MJ, Manley GT. Coagulopathy after traumatic brain injury. Neurosurgery 2012;70:1334-45. Full Text

5. Nekludov M, Antovic J, Bredbacka S, Blombäck M. Coagulation abnormalities associated with severe isolated traumatic brain injury: cerebral arteriovenous differences in coagulation and inflammatory markers. J Neurotrauma 2007;24:174-80. Full Text

6. McHugh GS, Engel DC, Butcher I, et al. Prognostic value of secondary insults in traumatic brain injury: results from the IMPACT study. J Neurotrauma 2007;24:287-93. Full Text

7. Reiff DA, Haricharan RN, Bullington NM, Griffin RL, McGwin G Jr, Rue LW 3rd. Traumatic brain injury is associated with the development of deep vein thrombosis independent of pharmacological prophylaxis. J Trauma 2009;66:1436-40. Full Text

8. Denson K, Morgan D, Cunningham R, et al. Incidence of venous thromboembolism in patients with traumatic brain injury. Am J Surg 2007;193:380-3. Full Text

9. Nathens AB, McMurray MK, Cuschieri J, et al. The practice of venous thromboembolism prophylaxis in the major trauma patient. J Trauma 2007;62:557-63. Full Text
10. Kim KS, Brophy GM. Symptomatic venous thromboembolism: incidence and risk factors in patients with spontaneous or traumatic intracranial hemorrhage. Neurocritical Care 2009;11:28-33. Full Text

11. Scales DC, Riva-Cambrin J, Wells D, Athaide V, Granton JT, Detsky AS. Prophylactic anticoagulation to prevent venous thromboembolism in traumatic intracranial hemorrhage: a decision analysis. Crit Care 2010;14(2):R72. Full Text

12. Scales DC, Riva-Cambrin J, Le TL, Pinto R, Cook DJ, Granton JT; Canadian Critical Care Trials Group. Prophylaxis against venous thromboembolism in neurointensive care patients: survey of Canadian practice. J Crit Care 2009;24:176-84. Full Text

13. Carlile MC, Yablon SA, Mysiw WJ, Frol AB, Lo D, Diaz-Arrastia R. Deep venous thrombosis management following traumatic brain injury: a practice survey of the traumatic brain injury model systems. J Head Trauma Rehabil 2006;21:483-90. Full Text

14. Dudley R, Aziz I, Bonnici A, et al. Early venous thromboembolic event prophylaxis in traumatic brain injury with low-molecular-weight heparin: risks and benefits. J Neurotrauma 2010;27:2165-72. Full Text

15. Minshall CT, Eriksson EA, Leon SM, Doben AR, McKinzie BP, Fakhry SM. Safety and efficacy of heparin or enoxaparin prophylaxis in blunt trauma patients with a head abbreviated injury severity score >2. J Trauma 2011;71:396-400. Full Text

16. Norwood SH, Berne JD, Rowe SA, Villarreal DH, Ledlie JT. Early venous thromboembolism prophylaxis with enoxaparin in patients with blunt traumatic brain injury. J Trauma 2008;65:1021-6. Full Text

17. Norwood SH, McAuley CE, Berne JD, et al. Prospective evaluation of the safety of enoxaparin prophylaxis for venous thromboembolism in patients with intracranial hemorrhagic injuries. Arch Surg 2002;137:696-701. Full Text

18. Guyatt GH, Akl EA, Crowther M, Gutterman DD, Schuünemann HJ; American College of Chest Physicians Antithrombotic Therapy and Prevention of Thrombosis Panel. Executive summary: Antithrombotic Therapy and Prevention of Thrombosis, 9th ed.: American College of Chest Physicians Evidence-Based Clinical Practice Guidelines. Chest 2012;141(2 Suppl):7S-47S. 